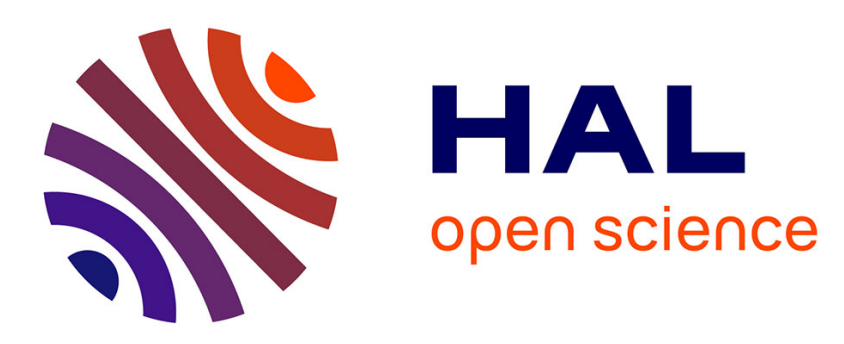

\title{
Full-Vector Gradient for Multi-spectral or Multivariate Images
}

\author{
Hermine Chatoux, Noël Richard, François Lecellier, Christine \\ Fernandez-Maloigne
}

\section{- To cite this version:}

Hermine Chatoux, Noël Richard, François Lecellier, Christine Fernandez-Maloigne. Full-Vector Gradient for Multi-spectral or Multivariate Images. IEEE Transactions on Image Processing, 2018, 10.1109/TIP.2018.2883794 . hal-02378882

\section{HAL Id: hal-02378882 \\ https://hal.science/hal-02378882}

Submitted on 25 Nov 2019

HAL is a multi-disciplinary open access archive for the deposit and dissemination of scientific research documents, whether they are published or not. The documents may come from teaching and research institutions in France or abroad, or from public or private research centers.
L'archive ouverte pluridisciplinaire HAL, est destinée au dépôt et à la diffusion de documents scientifiques de niveau recherche, publiés ou non, émanant des établissements d'enseignement et de recherche français ou étrangers, des laboratoires publics ou privés. 


\title{
Full-Vector Gradient for Multi-spectral or Multivariate Images
}

\author{
Hermine Chatoux $^{1}$, Noël Richard ${ }^{1}$, François Lecellier ${ }^{1}$ and Christine Fernandez-Maloigne ${ }^{1}$
}

\begin{abstract}
Gradient extraction is important for a lot of metrological applications such as Control Quality by Vision. In this work, we propose a full-vector gradient for multi-spectral sensors. The full-vector gradient extends Di Zenzo expression to take into account the non-orthogonality of the acquisition channels thanks to a Gram matrix. This expression is generic and independent from channel count. Results are provided for a color and a multi-spectral snapshot sensor. Then, we show the accuracy improvement of the gradient calculation by creating a dedicated objective test and from real images.
\end{abstract}

Index Terms-Gradient, multi-spectral, Gram matrix, Di Zenzo, multivariate, color.

\section{INTRODUCTION}

Thirty years after Di Zenzo's paper [1], the gradient estimation is still an open question for multi-spectral or multivariate images. The interdependencies between acquisition channels draw the limits of Di Zenzo's expression. Facing a lack of solutions in these domains, the existing surveys are dedicated to the comparison of gradient extraction in the visible and trichromatic color domain [2]-[4]. Furthermore, the multi-spectral and multivariate domains are not restricted to the visible range and to the sensitivity of the human visual system, they are more relative to the metrology and relationship between a physical acquired scene and the image content. Hence this work focuses on the gradient calculation in the acquisition space using the spectral sensitivity curves of the imaging system for metrological purposes in the optical and physical domain (Control Quality by Vision, Help to Diagnostic...).

\section{A. Related Work}

Existing approaches assess the gradient using ideas from Di Zenzo [1] or Canny [5]. A derivative form is measured from the discrete image. To avoid a direct derivation in the spatial domain, the images are usually convolved by a derivative form of a bi-dimensional function [6], [7]. Direct approaches process the gradient from each channel separately (marginal processing) before defining empirically a merging function to define the gradient norm and orientation (Shivakumara $e t$ al. [8]). Ehrhardt and Arridge in [9] use an inter-correlation between color channels specific to the image analyzed. Astola et al. [10] took the vector's nature into account by replacing the linear convolution with non-linear filtering. Some authors used this idea based on color distances [11]-[13]. Denis et al. [14] measured the gradient in the frequency domain using a quaternionic Fourier transform.

1 - Univ. Poitiers, XLIM, CNRS UMR 7252, F-86000 Poitiers, France
As pointed out by Rivest et al. [15], gradients are relative to high-spatial frequencies in images. These frequencies can be produced by noise or edges between image structures. Morphological gradients are defined to enhance pixel differences in a local neighborhood, avoiding the use of a derivative form on a non-continuous image. In addition, the extension to a multivariate case depends only on a color or spectral ordering [16]. Nevertheless, ordering often implies to select spectral or color references that impact the final result [17][19].

In [20], [21], the authors reduce the gradient to edges between two homogeneous regions through the analysis of local probability density functions. It can also be computed through a model requiring to solve partial derivative equations (Zareizadeh et al. [22]). The contributions from [23]-[25] ask a common question in the color domain: which color or multivariate space should be used to process the gradient? Some existing publications propose to stay in the acquisition space, so $R G B$ spaces for color images. Nevertheless, the spectral sensitivity functions of the sensor quite always overlap [26], [27]. Hence, corresponding acquisition channels are not independent, and the associated axis are not orthogonal. Since Carron and Lambert [28], lots of authors tried to transfer the problem in other color spaces, such as in Hue-based spaces where axis can be defined as semantically orthogonal (Lei $e t$ al. [29]). Unfortunately, the color transformation is defined by rotations from the initial $R G B$ acquisition space, that does not solve the problem of non-orthogonality, it only reduces its impact depending on the image content.

Several comparisons of gradient extraction from different color spaces conclude on the strong interest of CIELAB space [24], [25]. This perceptually uniform color space is well adapted to the perception variation relative to human vision (Saez et al. [30]). But Bello-Cerezo et al. [23] showed that $C I E L A B$ is more adapted when measurements required to change color spaces correctly are provided. To improve the gradient processing robustness to shadow or illumination changes, some color invariants are proposed [31]-[34]. On the other hand, Zhang et al. [35] use images edge detection before the image's demosaicing step.

Finally, when information about acquisition conditions are provided, the CIELAB color space is the most adapted. Otherwise, acquisition space fits better than other even with its non-orthogonality limit. The channel dependency has a direct impact on the $L_{2}$-norm's validity in acquisition space, and consequently, on the gradient norm [1], [36]. 


\section{B. Notation and content}

To lighten description of the different equations from this paper, we summarize the most used notations in table I.

In this work, we explain why the Di Zenzo expression implicitly considers the representation space as orthogonal (sec. II-B). Then, we present how to consider the channel interdependency in the gradient processing (sec. II-C). Two examples of color and multi-spectral domain adaptation are presented. The third section recalls the pre-processing steps allowing to compute a derivation from a non-continuous image in the color domain. Section IV presents the artificial database used in the result section. Two kinds of comparisons are presented, an objective one based on an accuracy criterion and generated images (sec. V-A and V-B) and a subjective one using real images from known databases (sec. V-C). Finally, we conclude in section VI.

\section{Complete Expression of Multivariate GRAdient}

Few articles explain each step of a color or spectral gradient extraction. We choose to start from the continuous domain to address the discrete and multivariate case next. Effectively, gradients are, most of the time, used as a black box. In this paper, we wish to explain every step from a mathematical and physical point of view.

Table I

NOTATIONS

\begin{tabular}{|c|c|}
\hline Notation & Meaning \\
\hline$x, y, z$ & Spatial location in $\mathbb{R}^{n}: x=\left(x_{1}, x_{2}\right)$ for a $2 D$ space. \\
\hline$a, b, c$ & $\begin{array}{l}\text { Color/multivariate coordinate in } \mathbb{R}^{m} \\
c=\left(c_{1}, c_{2}, c_{3}\right) \text { for a trichromatic color space. }\end{array}$ \\
\hline$s_{i}, S_{i}()$ & $\begin{array}{l}\text { Channel band } s_{i}, i \in[1, m] \\
\text { defined by its spectral sensitivity curve } S_{i}() \text {. }\end{array}$ \\
\hline$I, F, G$ & $\begin{array}{l}\text { Set or image, two definitions are considered: } \\
I: \mathbb{R}^{n} \rightarrow \mathbb{R}^{m} \quad I(x)=c, c \text { is a function; } \\
I \in \mathbb{R}^{n} \times \mathbb{R}^{m} \quad I(x, c) \text { a continuous map in } x \text { and } c .\end{array}$ \\
\hline $\mathcal{X}, \mathcal{C}, \mathcal{F}$ & $\begin{array}{l}\text { Support of the specified variable } x, c, f: \\
x \in \mathcal{X}=\left\{x_{i} \in\left[\operatorname{xmin}_{i} ; \max _{i}\right] ; i \in[1 ; n]\right\}\end{array}$ \\
\hline $\mathcal{S}(x)$ & Spatial neighborhood of $x$. \\
\hline$\# \mathcal{S}$ & Cardinal of mentioned set. \\
\hline$\|\cdot\|_{p}$ & $L_{p}$ norm (order-p Minkowski norm). \\
\hline$\langle., .\rangle_{p}$ & Scalar product associated to the corresponding $L_{p}$ norm. \\
\hline$d_{\Sigma}(.,)$. & Mahalanobis distance relative to the covariance matrix $\Sigma$. \\
\hline$[a]_{b}$ & $a$ Modulo $b$. \\
\hline$*$ & Convolution. \\
\hline \multicolumn{2}{|r|}{ Continuous space } \\
\hline$\frac{\partial I(a)}{\partial b}$ & $\begin{array}{l}\text { Partial derivative of } I(a) \text { with respect to } b \text { : } \\
\frac{\partial I\left(c_{2}\right)}{\partial x_{1}}: x_{1} \text { part of gradient measured at } c_{2} \text {. }\end{array}$ \\
\hline \multirow[t]{3}{*}{$\nabla I$} & $\begin{array}{l}\text { Gradient of the image } \mathrm{I} \text { : } \\
\text { for } I: \mathbb{R}^{2} \rightarrow \mathbb{R}^{3}\end{array}$ \\
\hline & \multirow{2}{*}{$\nabla I(x, c)=\left(\begin{array}{lll}\frac{\partial I\left(c_{1}\right)}{\partial x_{1}} & \frac{\partial I\left(c_{2}\right)}{\partial x_{1}} & \frac{\partial I\left(c_{3}\right)}{\partial x_{1}} \\
\frac{\partial I\left(c_{1}\right)}{\partial x_{2}} & \frac{\partial I\left(c_{2}\right)}{\partial x_{2}} & \frac{\partial I\left(c_{3}\right)}{\partial x_{2}}\end{array}\right)$} \\
\hline & \\
\hline \multicolumn{2}{|r|}{ Discrete space } \\
\hline$\Delta_{b} I(a)$ & $\begin{array}{l}\text { Partial derivative of } I(a) \text { with respect to } b \text { : } \\
\Delta_{x_{1}} I\left(c_{2}\right) \text { is the difference of two } c_{2} \text { along } x_{1} \text { direction. }\end{array}$ \\
\hline$\nabla I$ & $\nabla I(x, c)=\left(\begin{array}{lll}\Delta_{x_{1}} I\left(c_{1}\right) & \Delta_{x_{1}} I\left(c_{2}\right) & \Delta_{x_{1}} I\left(c_{3}\right) \\
\Delta_{x_{2}} I\left(c_{1}\right) & \Delta_{x_{2}} I\left(c_{2}\right) & \Delta_{x_{2}} I\left(c_{3}\right)\end{array}\right)$. \\
\hline
\end{tabular}

A differential is a linear function defined by equation (1). When applied to a vector, the differential provides the derivative along the vector's direction $p$.

$$
\lim _{\|h\|_{2} \rightarrow 0} \frac{\|I(p+h)-I(p)-d I(p) \cdot h\|_{2}}{\|h\|_{2}}=0,
$$

where $I: E \rightarrow F$ is a function ( $E$ and $F$ two vector spaces) and $d I$ the differential with $p, h \in E$. The derivative $d I(p)$ belongs to $F$, the multivariate or color domain, it is the differential of $I$ in $p$. Due to the limit definition $d I(p)$ is meaningful only close to $p$.

When measuring the differential of a basis vector of $E$, we obtain a partial derivative. Regrouping all partial derivatives in a vector is the gradient definition. In $2 \mathrm{D}$ gray level images, a basis is given by the derivative along $x_{1}$ and $x_{2}$ axis defining the gradient as a vector in $\mathbb{R}^{2}$. In multivariate cases, the vector is $2+m$ dimensional. Typically in the color case, the gradient is defined by:

$$
\nabla I(x, c)=\left(\begin{array}{l}
\frac{\partial I(x, c)}{\partial x_{1}} \\
\frac{\partial I(x, c)}{\partial x_{2}} \\
\frac{\partial I(x, c)}{\partial c_{1}} \\
\frac{\partial I(x, c)}{\partial c_{2}} \\
\frac{\partial I(x, c)}{\partial c_{3}}
\end{array}\right) .
$$

This gradient informs on the spatial and color direction of the image variations around $x$.

\section{A. Derivative adaptation to discrete context}

Equation (1) is defined for continuous functions. In a discrete domain, $h$ increments are limited to integer values, defining so the Roberts cross operator [37]:

$$
\begin{aligned}
& \Delta_{x_{1}} I(x)=I\left(x_{1}+1, x_{2}\right)-I\left(x_{1}, x_{2}\right), \\
& \Delta_{x_{2}} I(x)=I\left(x_{1}, x_{2}+1\right)-I\left(x_{1}, x_{2}\right) .
\end{aligned}
$$

To avoid the asymmetry induced by the previous expression, even values of $h$ are preferred, starting with $h=2$ :

$$
\begin{aligned}
& \Delta_{x_{1}} I(x)=I\left(x_{1}+1, x_{2}\right)-I\left(x_{1}-1, x_{2}\right), \\
& \Delta_{x_{2}} I(x)=I\left(x_{1}, x_{2}+1\right)-I\left(x_{1}, x_{2}-1\right) .
\end{aligned}
$$

\section{B. Adaptation to a theoretical multivariate context}

Di Zenzo was the first to propose a color extension to gradient processing considering color space as a vector space [1]. Koschan and Abidi [36] introduced the same idea with a physical approach whereas Di Zenzo has a mathematical one. Both papers study color information in a spatial autocorrelation matrix:

$$
A C o r=\left(\begin{array}{cc}
\left\|\frac{\partial I(x, c)}{\partial x_{1}}\right\|_{2}^{2} & \left\langle\frac{\partial I(x, c)}{\partial x_{1}}, \frac{\partial I(x, c)}{\partial x_{2}}\right\rangle_{2} \\
\left\langle\frac{\partial I(x, c)}{\partial x_{1}}, \frac{\partial I(x, c)}{\partial x_{2}}\right\rangle_{2} & \left\|\frac{\partial I(x, c)}{\partial x_{2}}\right\|_{2}^{2}
\end{array}\right),
$$


with

$$
\begin{array}{r}
\left\|\frac{\partial I(x, c)}{\partial x_{i}}\right\|_{2}^{2}=\left\|\frac{\partial I\left(x, c_{1}\right)}{\partial x_{i}}\right\|_{2}^{2}+\left\|\frac{\partial I\left(x, c_{2}\right)}{\partial x_{i}}\right\|_{2}^{2}+\left\|\frac{\partial I\left(x, c_{3}\right)}{\partial x_{i}}\right\|_{2}^{2} \\
\text { and }\left\langle\frac{\partial I(x, c)}{\partial x_{1}}, \frac{\partial I(x, c)}{\partial x_{2}}\right\rangle_{2}= \\
+\frac{\partial I\left(x, c_{1}\right)}{\partial x_{1}} \frac{\partial I\left(x, c_{1}\right)}{\partial x_{2}} \\
+\frac{\partial I\left(x, c_{2}\right)}{\partial x_{1}} \frac{\partial I\left(x, c_{2}\right)}{\partial x_{2}} \\
+\frac{\partial I\left(x, c_{3}\right)}{\partial x_{1}} \frac{\partial I\left(x, c_{3}\right)}{\partial x_{2}} .
\end{array}
$$

The theoretical $R G B$ color space is orthogonal, but in reality, $R G B$ color spaces are not. Di Zenzo implicitly considers the scalar product between channels to be null. The complete norm should be:

$$
\left\|\frac{\partial I(x, c)}{\partial x_{i}}\right\|_{2}^{2}=\sum_{j=1}^{3} \sum_{k=1}^{3}\left\langle\frac{\partial I\left(x, c_{j}\right)}{\partial x_{i}}, \frac{\partial I\left(x, c_{k}\right)}{\partial x_{i}}\right\rangle_{2} .
$$

The absence of cross product in Di Zenzo's formulation implies the orthogonal hypothesis on the representation space axis.

To estimate the gradient's norm, the eigenvalues of the autocorrelation matrix are used. Different expressions were proposed, Sapiro [38] expressed, for example, the gradient magnitude $M a g$ as the difference between the two eigenvalues:

$$
\begin{aligned}
M a g^{2}= & \left(\left\|\frac{\partial I(x, c)}{\partial x_{1}}\right\|_{2}^{2}-\left\|\frac{\partial I(x, c)}{\partial x_{2}}\right\|_{2}^{2}\right)^{2} \\
& +4\left\langle\frac{\partial I(x, c)}{\partial x_{1}}, \frac{\partial I(x, c)}{\partial x_{2}}\right\rangle_{2}^{2}
\end{aligned}
$$

The spatial direction $\theta$ of the gradient, defined by Jin et al. [39], lifts the imprecision of $\pm \frac{\pi}{2}$ from the initial Di Zenzo expression:

$$
\theta=\left\{\begin{array}{l}
\text { Undefined if }\left(\left\|\frac{\partial I(x, c)}{\partial x_{1}}\right\|_{2}^{2}-\left\|\frac{\partial I(x, c)}{\partial x_{2}}\right\|_{2}^{2}\right)^{2}+(\tau)^{2}=0 \\
\operatorname{sign}(\tau) \arcsin \left(\frac{\beta_{+}-\left\|\frac{\partial I(x, c)}{\partial x_{1}}\right\|_{2}^{2}}{2 \beta_{+}-\left\|\frac{\partial I(x, c)}{\partial x_{1}}\right\|_{2}^{2}-\left\|\frac{\partial I(x, c)}{\partial x_{2}}\right\|_{2}^{2}}\right) \\
\text { with } \tau=\left\langle\frac{\partial I(x, c)}{\partial x_{1}}, \frac{\partial I(x, c)}{\partial x_{2}}\right\rangle_{2} \text { else }
\end{array}\right.
$$

where $\beta_{+}$is the maximum eigenvalue.

\section{Adaptation to non-independent channel context}

Color sensors and a great majority of multi-spectral sensors are constructed with overlapping spectral sensitivity functions as shown in figures 1 or 2 . Therefore, there is a correlation between channels and the classical scalar product in $3 D$ is not suitable. To take care of the color spaces non-orthogonality, the scalar product between $I(x)$ and $I(y)$ must be defined with the Gram matrix (eq. (12)). It is a matrix computed from the scalar product of each basis element [40], [41]. Equation (11) is normalized in reference to white balance.

$$
\langle I(x), I(y)\rangle=\frac{I(x)^{T} \cdot G m \cdot I(y)}{\|G m\|_{\infty}},
$$

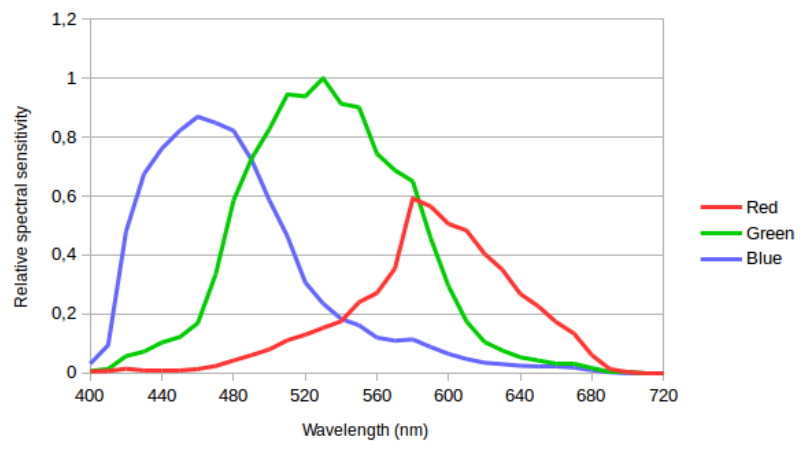

Figure 1. Spectral sensitivity functions of the camera Canon 500D.

$$
G m=\left(\begin{array}{cccc}
\left\|s_{0}\right\|_{2}^{2} & \left\langle s_{0}, s_{1}\right\rangle_{2} & \ldots & \left\langle s_{0}, s_{m}\right\rangle_{2} \\
\left\langle s_{1}, s_{0}\right\rangle_{2} & \left\|s_{1}\right\|_{2}^{2} & \ldots & \left\langle s_{1}, s_{m}\right\rangle_{2} \\
\vdots & \vdots & \ddots & \vdots \\
\left\langle s_{m}, s_{0}\right\rangle_{2} & \ldots & \left\langle s_{m}, s_{m-1}\right\rangle_{2} & \left\|s_{m}\right\|_{2}^{2}
\end{array}\right)
$$

Scalar products in the Gram matrix are supposed to be our initial basis decomposed in an orthogonal one. No orthogonal basis is known in an $R G B$ color space. Therefore, we need a different scalar product defined in the spectral domain. We use the spectral sensitivity functions $S_{i}()$ and $S_{j}()$ of each channel. The Gram matrix coefficients are defined by:

$$
\left\langle s_{i}, s_{j}\right\rangle_{2}=\int_{\mathbb{R}} S_{i}(\lambda) S_{j}(\lambda) d \lambda .
$$

1) Application in color domain: considering a real case, using a Canon 500D photographic sensor, spectral sensitivity functions can be obtained by experimental measures or directly from the constructor. In figure 1, all three spectral sensitivity functions $\bar{r}(), \bar{g}()$ and $\bar{b}()$ show strong correlations between channels. In such case, Gram matrix is reduced to

$$
\begin{aligned}
G m= & \left(\begin{array}{ccc}
\|R\|_{2}^{2} & \langle R, G\rangle_{2} & \langle R, B\rangle_{2} \\
\langle R, G\rangle_{2} & \|G\|_{2}^{2} & \langle G, B\rangle_{2} \\
\langle R, B\rangle_{2} & \langle G, B\rangle_{2} & \|B\|_{2}^{2}
\end{array}\right), \\
\text { with } \quad\langle R, G\rangle_{2}=\int_{\lambda_{\min }}^{\lambda_{\max }} r(\lambda) g(\lambda) d \lambda, &
\end{aligned}
$$

where $\lambda_{\min }=360 \mathrm{~nm}$ and $\lambda_{\max }=780 \mathrm{~nm}$, the CIE standard limits of the visible range. This color sensor Gram matrix is given in equation (16).

$$
G m_{\text {Canon } 500 D}=\left(\begin{array}{lll}
0.140 & 0.166 & 0.047 \\
0.166 & 0.566 & 0.268 \\
0.047 & 0.268 & 0.388
\end{array}\right)
$$

We can see the matrix is far from diagonal. The integral of the red sensitivity curves is smaller than for the green, which explains the different coefficient on the diagonal. Due to a small overlapping, the red and blue curves present a small correlation factor of 0.047 . Blue and green overlap more, hence a higher correlation 0.268 .

In a more generic context, when the spectral sensitivity functions of the sensor are unknown, the CIE sensitivity 


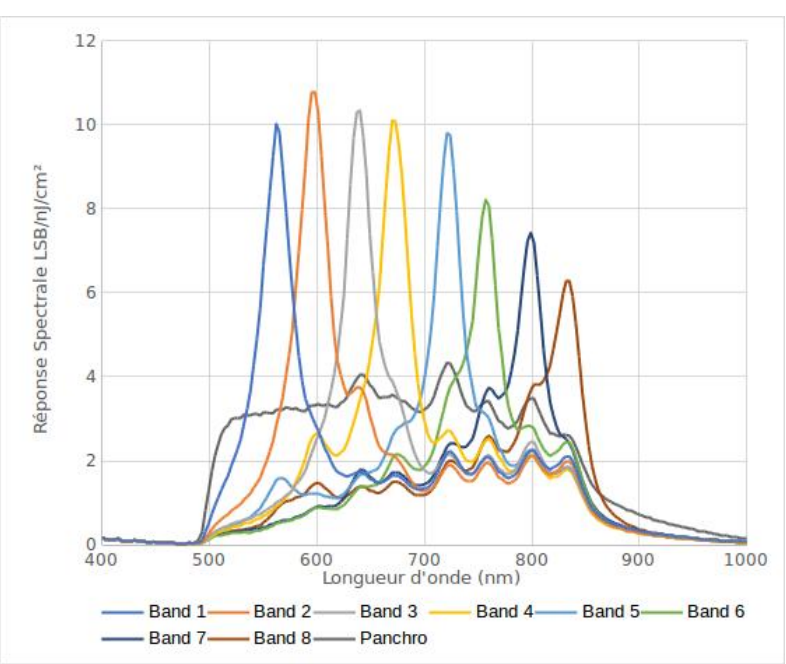

Figure 2. Spectral sensitivity functions of the multi-spectral snapshot camera CMS-V (SILIOS Technologies) with eight spectral bands and one panchromatic channel (CMS-C $3 \times 3$ matrix).

curves can be used as an approximation. Since we work with synthetic dataset and color images without known calibration information, we will consider the $C I E R G B$ as the acquisition space and use the $C I E$ sensitivity curves obtained for observations at $10^{\circ}$ of visual angle for our experiments. As a physical sensor cannot have negative values, we have added a constant to the CIERGB sensitivity curves to only have positive values. The Gram matrix obtained in this case is:

$$
G m_{C I E R G B}=\left(\begin{array}{lll}
0.623 & 0.227 & 0.150 \\
0.227 & 0.183 & 0.117 \\
0.150 & 0.117 & 0.129
\end{array}\right) .
$$

Contrary to $G m_{C a n o n 500 D}$, the $G m_{C I E R G B}$ red coefficients (first row and column) are high due to the correlation of the $C I E R G B$ red sensitivity curves with the blue and green ones. Overlapping between blue and green curves is weak which explains smaller coefficients.

To use the full-vector gradient with another acquisition space, one only needs the sensitivity curves to estimate the Gram matrix associated to the sensor. It could be adapted to a display device but this asks another question that needs new perceptual experiments and a whole new scientific paper.

2) Application to the Multi-Spectral Domain: the proposed expression is fully generic and can be directly adapted for different multivariate cases. To illustrate this, we extend it to the multi-spectral domain for a 9-bands sensor (fig. 2). The first eight spectral filters are centered respectively at $\{561,596,638,673,722,758,801,838\} \mathrm{nm}$. The last one is a panchromatic filter. Knowing the spectral sensitivity curves from the sensor constructor, The Gram matrix is directly deduced from the measurements:

$\left(\begin{array}{lllllllll}0.141 & 0.107 & 0.073 & 0.072 & 0.073 & 0.058 & 0.057 & 0.058 & 0.115 \\ 0.107 & 0.168 & 0.107 & 0.095 & 0.073 & 0.062 & 0.061 & 0.064 & 0.122 \\ 0.073 & 0.107 & 0.149 & 0.115 & 0.079 & 0.070 & 0.070 & 0.063 & 0.119 \\ 0.072 & 0.095 & 0.115 & 0.149 & 0.094 & 0.079 & 0.071 & 0.064 & 0.118 \\ 0.073 & 0.073 & 0.079 & 0.094 & 0.130 & 0.092 & 0.074 & 0.065 & 0.112 \\ 0.058 & 0.062 & 0.070 & 0.079 & 0.092 & 0.104 & 0.081 & 0.068 & 0.095 \\ 0.057 & 0.061 & 0.070 & 0.071 & 0.074 & 0.081 & 0.091 & 0.072 & 0.089 \\ 0.058 & 0.064 & 0.063 & 0.064 & 0.065 & 0.068 & 0.072 & 0.077 & 0.082 \\ 0.115 & 0.122 & 0.119 & 0.118 & 0.112 & 0.095 & 0.089 & 0.082 & 0.148\end{array}\right)$

To comment on this Gram matrix, first, we note that diagonal values are higher than others. Channels corresponding to the red and near infrared present a reduced sensitivity, their corresponding values are smaller than values corresponding to channels in the lowest wavelengths. Secondly, we observe the relative importance of all the values for the panchromatic filter on the last column and row. It is explained by the shape of this filter (gray on fig. 2). Thirdly, the sub-diagonal present higher correlation than the rest of the matrix. When looking at figure 2, we observe a filter (except the panchromatic one) overlaps mostly with only two other filters: the previous and the next ones.

\section{Color AND Discrete AdAPtation}

In this section, we raise the problem of discrete function derivation. Indeed, these functions require low-pass filtering. Secondly, we will present Carron's gradient which takes into account inter-channel dependency in $H L S$ color space.

\section{A. Toward $C^{1}$-Class Function}

Mathematically, to estimate a gradient, a function must be differentiable and the derivative has to be continuous. In other words, it must be a $C^{1}$-class function. By construction, digital images are not $C^{0}$ [15], thus a low-pass filter is required to satisfy the constraint.

Sobel and Feldman in [42], then Prewitt in [43] were the first to propose low-pass filtering in the orthogonal direction of gradient extraction. In both cases, the authors use symmetrical gradient estimation $(h=2)$. Prewitt filter is constructed from a basic average filter. Sobel uses a filter approaching Gaussian coefficients. It offers better filtering performances. For example, the $3 \times 3$ Sobel filter is given by:

$$
\begin{aligned}
\frac{\partial I(x)}{\partial x_{1}} & =\left(\begin{array}{ccc}
-1 & 0 & 1 \\
-2 & 0 & 2 \\
-1 & 0 & 1
\end{array}\right) * I(x), \\
\text { and } \frac{\partial I(x)}{\partial x_{2}} & =\left(\begin{array}{ccc}
-1 & -2 & -1 \\
0 & 0 & 0 \\
1 & 2 & 1
\end{array}\right) * I(x) .
\end{aligned}
$$

Later, Canny [5] proposed to use a bi-dimensional Gaussian filter (eq. (20)) before the gradient computation in order to obtain a $C^{1}$-class function.

$$
G(I(x))=\frac{1}{\sigma \sqrt{2 \pi}} e^{\frac{-x^{2}}{2 \sigma^{2}}} .
$$

\section{B. Adaptation to the color specificities}

The choice of color space is always important and crucial in color image processing. In 2014, Wang et al. [24] compared different color spaces for segmentation purposes and concluded that $C I E L A B$ is the most robust one. Bello-Cerezo et al. [23] experimented on the same question for classification purposes. The authors are more precise as their interest lies in existing or estimated calibration information. The color axis orthogonality of $C I E L A B$ space and the perceptual distance validity allow asserting the $C I E L A B$ superiority for material classification. Nevertheless, the bias introduced by bad calibration information reduces performances compared to those 
obtained in $R G B$ spaces. In response to this problem, Carron proposed in 1994 to decompose the gradient calculation in $H L S$ space, supposed orthogonal [28].

\section{Taking Channel Interdependency in HLS Color Space}

$H L S$ is not a Euclidean space and has cylindrical coordinates. Therefore, Carron defined the gradient as a weighted combination of marginal derivatives extracted in $H L S$ color space. The weighted combination takes into account saturation magnitude, to set the hue information relevance. The proposed expression uses fuzzy logic to obtain a behavior close to human vision. The hue gradient is defined as:

$\Delta(I(x, H), I(y, H))=p(I(x, S), I(y, S))[I(x, H)-I(y, H)]_{\pi}$,

with

$$
p(I(x, S), I(y, S))=\sqrt{\alpha(I(x, S)) \cdot \alpha(I(y, S))},
$$

and $\alpha(S)$ being a function giving relevance to the hue when saturation is high. Finally, the three derivatives are combined accordingly:

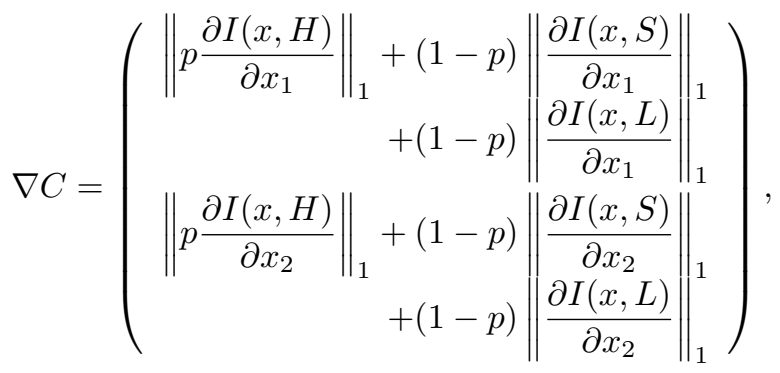

where $p$ is the coefficient of equation (22).

For the experiment, a selected constraint for the gradient is to manage the channel dependencies. Thus, we will compare the full vector gradient with Di Zenzo's gradient computed in the acquisition space. We add to this list the Carron gradient processed in $I H L S$ space [44].

\section{Complete expression of the full-vector gradient}

In this section, we give the complete expression of the fullvector gradient (FVG), starting from the Di Zenzo construction expressed through the spatial autocorrelation matrix (eq. (24)).

$$
A C o r=\left(\begin{array}{l}
\left\langle\frac{\partial I(x, c)}{\partial x_{1}}, \frac{\partial I(x, c)}{\partial x_{1}}\right. \\
\left\langle\frac{\partial I(x, c)}{\partial x_{1}}, \frac{\partial I(x, c)}{\partial x_{2}}\right\rangle
\end{array}\right\rangle \quad\left\langle\begin{array}{l}
\frac{\partial I(x, c)}{\partial x_{1}}, \frac{\partial I(x, c)}{\partial x_{2}} \\
\left.\frac{\partial I(x, c)}{\partial x_{2}}, \frac{\partial I(x, c)}{\partial x_{2}}\right\rangle
\end{array}\right\rangle .
$$

To define this gradient, we need to express the complete scalar product. To take into account the non-orthogonality of the representation space, the scalar products inside the autocorrelation matrix ACor must be defined using the Gram matrix (eq. (12)):

$$
\left\langle\frac{\partial I(x, c)}{\partial x_{k}}, \frac{\partial I(x, c)}{\partial x_{l}}\right\rangle=\frac{\left(\frac{\partial I(x, c)}{\partial x_{k}}\right)^{T} G_{m}\left(\frac{\partial I(x, c)}{\partial x_{l}}\right)}{\left\|G_{m}\right\|_{\infty}} .
$$

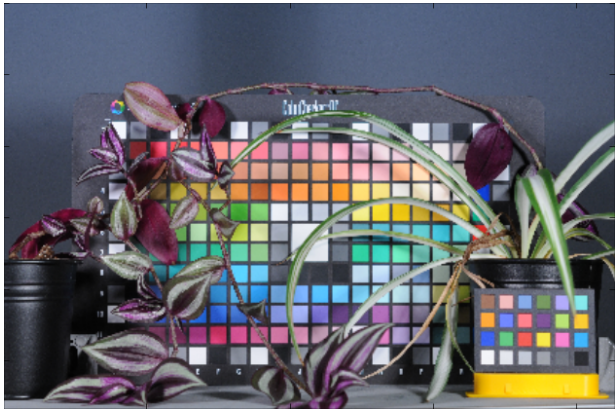

(a) Image taken with a Canon 500D

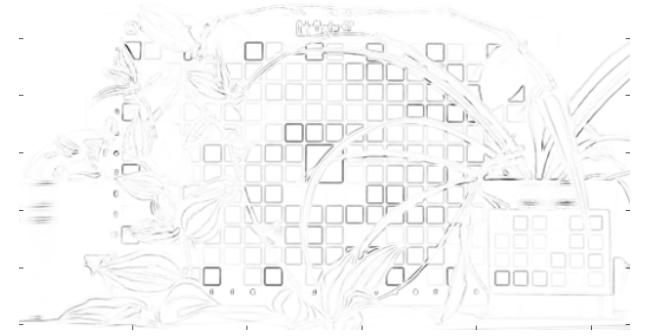

(b) Full-vector gradient

Figure 3. Example of the full-vector gradient with an image from a Canon 500D.

The scalar product associated with the Gram matrix is measured with the spectral sensitivity curves $S_{i}()$ corresponding to each sensor's channel $s_{i}$ :

$$
\left\langle s_{i}, s_{j}\right\rangle_{2}=\int_{\lambda_{\min }}^{\lambda_{\max }} S_{i}(\lambda) S_{j}(\lambda) d \lambda .
$$

Due to the optical filter before the sensor and to the sensor limits, the integral bounds are a lower wavelength and an upper wavelength.

The gradient magnitude is then defined directly from the Di Zenzo expression and the scalar product defined in equation (25):

$$
\begin{aligned}
M^{2} g^{2}= & \left(\left\langle\frac{\partial I(x, c)}{\partial x_{1}}, \frac{\partial I(x, c)}{\partial x_{1}}\right\rangle-\left\langle\frac{\partial I(x, c)}{\partial x_{2}}, \frac{\partial I(x, c)}{\partial x_{2}}\right\rangle\right)^{2} \\
& +4\left\langle\frac{\partial I(x, c)}{\partial x_{1}}, \frac{\partial I(x, c)}{\partial x_{2}}\right\rangle^{2}
\end{aligned}
$$

Figures 3 and 4 give an acquisition of the same scene for a Canon 500D and a SILIOS CMS-V camera and the calculation of the full-vector gradient on these acquisitions. Figures $3 \mathrm{~b}$ and $4 \mathrm{~d}$ should not be compared. Firstly, because the two sensors have very different spatial resolutions. Secondly, the multi-spectral sensors do not perceive blue wavelength but infrared ones. Finally, the sensors sizes are different, as the cameras objectives. That explains the difficulty to compare both images.

\section{Proposed Protocol}

The second contribution of this article is to propose an objective test for gradient performance assessment. Facing a 


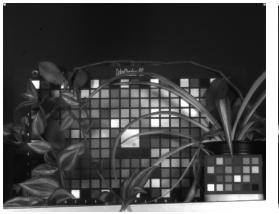

(a) CMS-V channel 1

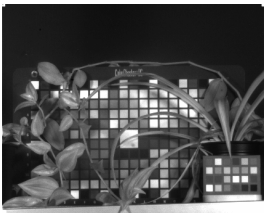

(b) CMS-V channel 6

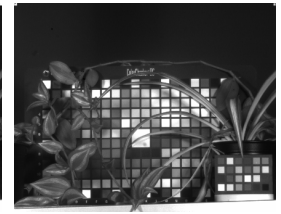

(c) CMS-V panchromatic channel

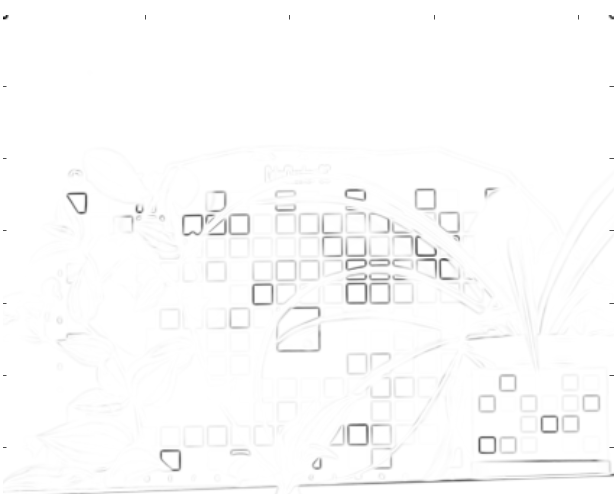

(d) Full-vector gradient
Figure 4. Example of the full-vector gradient with a multi-spectral image from a CMS-V camera (SILIOS Technologies).

lack of existing and reference results in the multi-spectral domain to compare the proposed approach, we choose to develop this assessment in the trichromatic color domain. As the proposed gradient is generic, there is no necessity to assess its validity in every possible spectral/multivariate case. Secondly, gradient assessment is widely developed in color domain, with an important literature allowing to compare our approach to existing ones.

We will, first, describe our motivations, then how color distributions are created. Then, we will present the separability rate that allows us to measure the difficulty to extract an edge. Thanks to the separability rate and the color distribution shapes, we have an artificial dataset. Finally, the comparison criterion based on classification is presented.

\section{A. Motivations}

Magnier et al. propose a gray-level gradient quality assessment on real images in [45]. As ground truth depends on a human performing segmentation, we have decided to create an artificial dataset.

We assume it is easy to perform a gradient detection when both regions are two uniform colors, even if the color difference is low. But when the regions are not perfectly uniform, they are defined by their respective color distributions. The central idea of this dataset is to consider each side of the edge as a color distribution. The gradient extraction complexity is relative to the intersection of the two color distributions. The more they overlap, the more difficult it is for the gradient to segment correctly.

The proposed protocol is based on two color distributions generated with a given separability rate $r$, distribution shapes and color averages (figure 5).

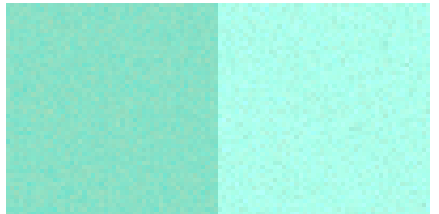

(a) $r=0.5, C_{1}=(85 ;-47 ; 7)$, $C_{2}=(96 ;-42 ; 5)$.

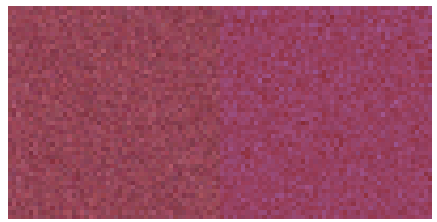

(c) $r=0, C_{1}=(38 ; 52 ; 3)$, $C_{2}=(37 ; 60 ;-7)$.

Figure 5. Examples of generated images. The separability rate values $(r)$ and the color pairs location is given per images. The color difference between the distribution center is fixed to 12 to perceive differences.

\section{B. Color Distribution Generation}

The generated color distribution is defined using a multivariate Gaussian distribution $\mathcal{N}_{3}(\mu, \Sigma)$ in a given color space $(C I E R G B)$. To avoid bias due to quantification step, values are kept in double precision.

The multivariate Gaussian distribution structure is controlled by the variance-covariance matrix $\Sigma$, a rotation matrix $R$ and a shape matrix $S$ (eq. (28)). A scalar parameter $a$ allows controlling the distribution shape, spherical when $a$ tends to 1 , or oblong when $a$ tends to 5 (eq. (29)). This parameter is randomly defined in the experiment. The $R$ matrix is a classical rotation matrix defined by three angles $\theta$, also randomly selected.

$$
\begin{aligned}
\Sigma= & R^{T} S R, \\
& \text { with } S=\left(\begin{array}{ccc}
a & 0 & 0 \\
0 & \frac{1}{a} & 0 \\
0 & 0 & \frac{1}{a^{2}}
\end{array}\right) .
\end{aligned}
$$

Figure 6 shows some examples of obtained shapes. At this level, all distributions are centered and normalized $\left(\mathcal{N}_{3}(0, \Sigma)\right)$. The ellipsoids represent the distribution's hull at a Mahalanobis distance of 3 from the center.

Then, we modify their means and add a scalar parameter $\alpha$ to control the volume of the distribution. The final covariance matrix is then defined by $\Sigma_{i}^{\prime}=\alpha_{i}^{2} \Sigma_{i}$. This step allows us to control the distributions overlap. Two centers are randomly selected $C_{1}$ and $C_{2}$ in $C I E L A B$ to be at a $\triangle E$ color difference of 3 .

\section{Separability rate}

The gradient detection complexity is proportional to the intersection of the two three-dimensional ellipsoids relative to the multivariate Gaussian distributions $\mathcal{N}_{3}\left(C_{1}, \Sigma_{1}^{\prime}\right)$ and $\mathcal{N}_{3}\left(C_{2}, \Sigma_{2}^{\prime}\right)$.

We propose to use the normalized algebraic distance between the two color distribution hulls (eq. (30)) to define the separability rate $(r)$ as illustrated in figure 7 . The hull of the 


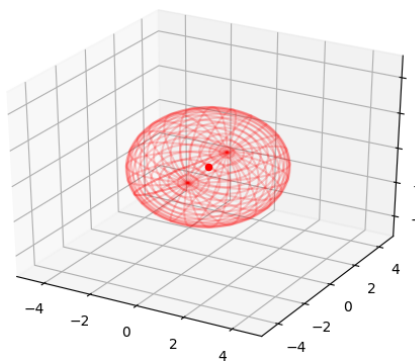

(a) $a=1.0, \theta=(247 ; 49 ; 162)$

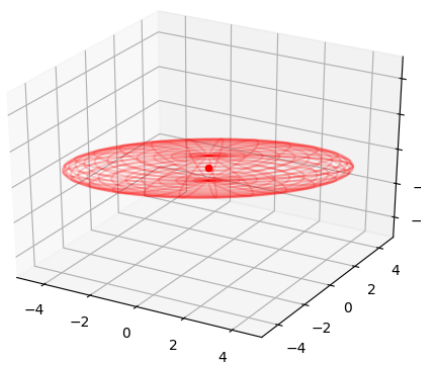

(c) $a=3.0, \theta=(282 ; 117 ; 16)$

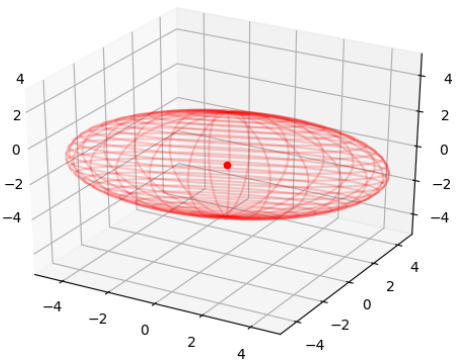

(d) $a=3.9, \theta=(9 ; 110 ; 275)$

Figure 6. Examples of distribution hull at a Mahalanobis distance of 3 units from the center of the generated distributions.

considered color distribution is defined by the ellipsoid surface at a Mahalanobis distance of 3 units from the distribution center. The value of 3 units allows containing more than $99 \%$ of the sample data inside the ellipsoid hull.

$$
\begin{array}{ll}
r=\frac{\overline{C_{1}^{\prime} C_{2}^{\prime}}}{\left\|C_{1} C_{2}\right\|_{2}} \quad \text { with } \quad d_{\Sigma_{i}^{\prime}}\left(C_{i}, C_{i}^{\prime}\right)=3, \\
\text { and } \quad\left\{C_{1}^{\prime}, C_{2}^{\prime}\right\} \in\left(C_{1} C_{2}\right) .
\end{array}
$$

A null value of the separability rate $r$ expresses a single intersection point between the two ellipsoids. The two distributions overlap on less than $1 \%$ of color pixels. A negative value of $r$ expresses a more important intersection, in opposition to a positive value that shows an empty intersection of the ellipsoids (but distributions can slightly overlap). The normalization is relative to the distance between the distribution center. This normalization allows comparison between different color center differences and/or different color spaces.

Another possibility for the separability rate could be the Kullback-Leibler divergence (sometimes called Jeffrey's divergence). Obviously, the non-linear nature ${ }^{1}$ of this kind of similarity measures makes the dynamics of the results more difficult to interpret.

\section{The artificial dataset}

To explore all possible colors in the experiment, color pairs $\left(C_{1}, C_{2}\right)$ are randomly chosen. Both distribution magnification rates $\alpha_{i}$ are calculated given the separability rate $r$ :

$$
\alpha=\alpha_{1}=\alpha_{2}=\frac{(1-r) d_{\Sigma_{1}}\left(C_{1}, C_{2}\right) d_{\Sigma_{2}}\left(C_{2}, C_{1}\right)}{3\left(d_{\Sigma_{1}}\left(C_{1}, C_{2}\right)+d_{\Sigma_{2}}\left(C_{2}, C_{1}\right)\right)} .
$$

\footnotetext{
${ }^{1}$ The Kullback-Leibler divergence of a multivariate Gaussian distribution is easy to produce but shows a square relationship to the distance centers and a $\log$ difference of the variance-covariance matrices.
}

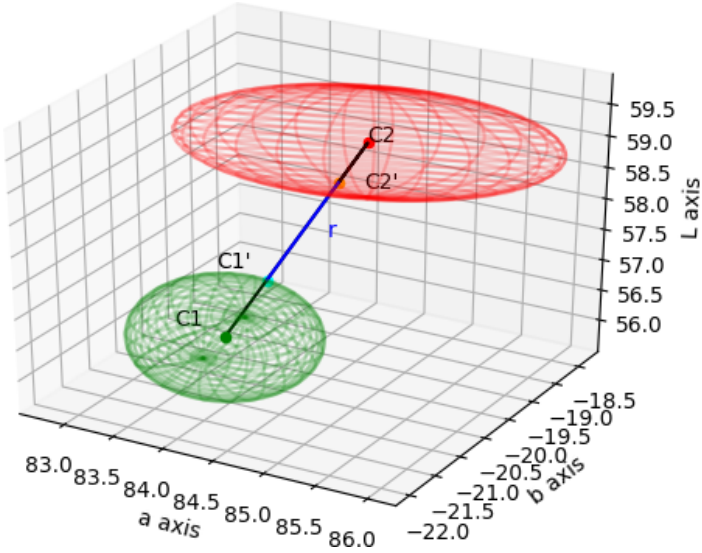

(a) Distributions 6a (green) and 6d (red) with $r=0.5$

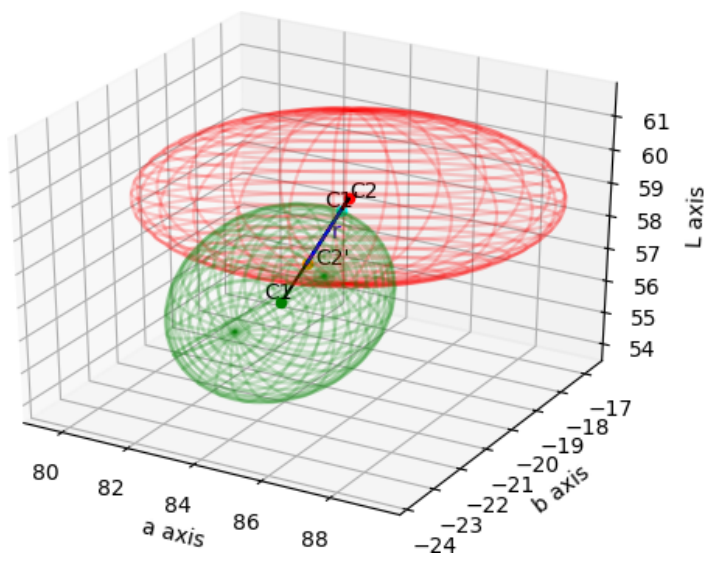

(b) Distributions 6a (green) and 6d (red) with $r=-0.5$

Figure 7. Illustration of the separability rate $(r)$ depending on the overlap between two color distributions.

The separability rate explores the range $[-1,+1]$. In addition, we verify that both color distributions belong to the $C I E R G B$ color gamut. Color distributions are then generated in $C I E R G B$.

In figure 5, we present four generated images with two pairs of color $C_{i}$. In order to see the color difference between the two regions defined by their color distributions, we selected a color difference $\Delta E\left(C_{1}, C_{2}\right)=12$. Parameters of the distributions in figures $5 \mathrm{a}$ and $5 \mathrm{~b}$ induce a difference mainly at a luminance level. On the contrary, the other two figures differ at a hue level. The separability rate on figure $5 \mathrm{~b}$ (resp. 5d) is higher than figure $5 \mathrm{a}$ (resp. 5c), because the distributions overlap more. They are trickier to segment.

\section{E. Criteria of Comparison}

To assess the quality of gradients, we will segment the images and use a criterion initially exploited in data classification assessment. Each test image is composed of two rectangular regions $R_{1}$ and $R_{2}$ (fig. 5 presents images $(\mathcal{I}$ ) where gradients will be measured). Our objective is to evaluate our channel interdependency management and not the edge direction. Thus, we have chosen to focus on one vertical edge. Edge location is based on the maximal norm of the computed gradient per row. True positive $(\mathcal{T P}$ ) pixels (resp. true negative $\mathcal{T N}$ ) are pixels affected after the edge detection to the $R_{1}$ side 
(resp. $R_{2}$ side) and initially located in the $R_{1}$ region (resp. $R_{2}$ region). False positive $(\mathcal{F P})$ and negative $(\mathcal{F} \mathcal{N})$ pixels correspond to pixels that are located on the wrong side of the edge after gradient processing. Figure 8 shows a theoretical segmentation and a practical one for $3 \times 3$ regions. We use the accuracy criteria to evaluate performances:

$$
A c c=\frac{\# \mathcal{T} \mathcal{P}+\# \mathcal{T} \mathcal{N}}{\# \mathcal{I}} .
$$

Accuracy results are expected to be between $0 \%$ and $100 \%$, $100 \%$ being the perfect response.

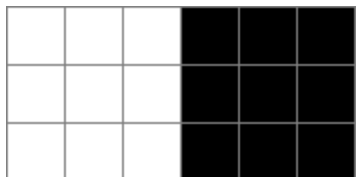

(a) Theoretical segmentation

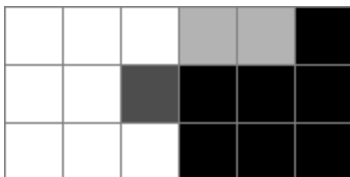

(b) Example of practical result
Figure 8. Example of practical segmentation compared to the theoretical case. In $8 \mathrm{~b}$, white is true positive, light gray is false positive, dark gray is false negative and black is true negative compared to 8a. The accuracy in this example is of $\frac{8+7}{18}$

\section{RESUlts AND Discussion}

In this section, we compare the edge extraction accuracy using a gradient computed from synthetic and real images. The first two experiments use the artificial dataset, the first one assessing the impact of the color distribution shapes on the edge detection performances, and the second assessing the global performances. The third one compares the gradient detection of real world images (Stex [46] and VisTex [47] database).

\section{A. Impact of Color Distribution Shape}

In the real world, the color distribution shapes are not always spherical. This experiment assesses the impact of the distribution shape on the edge extraction performances. Four different shapes are defined (fig. 6) allowing to create 16 pairs of color distributions. In each case, 10 pairs of color were used to create color images. Figure 9 presents the average from the 10 color pairs at each separability rate in the case of four relevant pairs of distribution shapes (the other twelve present similar results).

Results from figure 9a evaluate the edge extraction from regions generated with almost spherical color distributions, so without channel interdependency. As expected the Di Zenzo approach presents good performances followed closely by the full-vector gradient approach. In the second result, one of the two distributions is slightly ellipsoidal and oriented (fig. 9b). The Di Zenzo approach becomes less efficient. This result is reinforced when the second distribution is more oblong (fig. 9c). Figure 9d illustrates the results obtained in a more generic case: two ellipsoidal and oriented distributions. In this last case, the limits of marginal and Di Zenzo's approaches are reached.

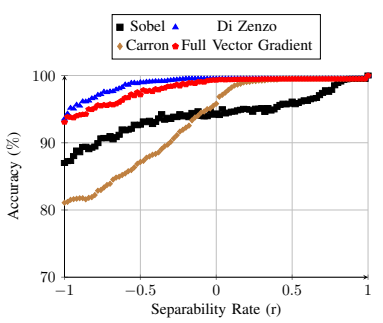

(a) Distributions $6 a$ and $6 a$

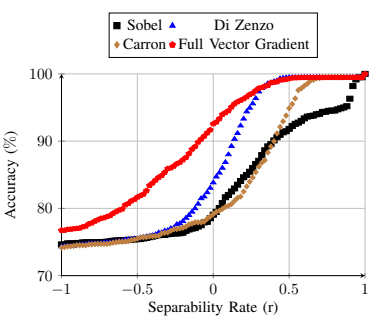

(c) Distributions $6 a$ and $6 \mathrm{~d}$

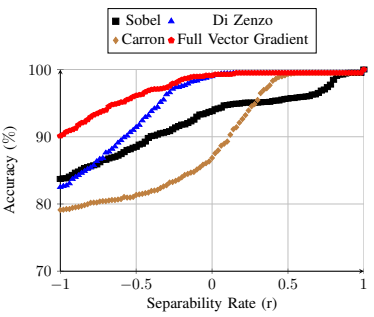

(b) Distributions $6 \mathrm{a}$ and $6 \mathrm{~b}$

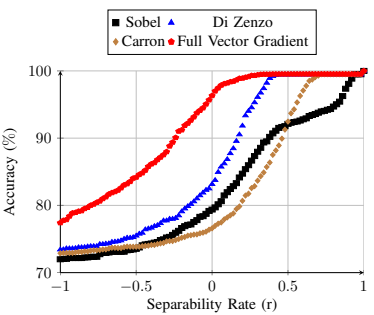

(d) Distributions 6c and 6b
Figure 9. Examples of edge detection accuracy assessment for images generated with $\Delta E=3$. Only two distributions are used per results. The shape of these distributions is given per sub figure and refers to figure 6 .

\section{B. Combining Different Color Distribution Shapes}

To compare results on a larger scale, we raise the number of cases using 500 images for each separability rate. Fifty distribution shapes for the images left part and 50 others for the right with 10 color pairs. We estimated the average accuracy for eight separability rates (tab. II).

The full-vector gradient performs better than the other gradients. The differences are less important than those observed previously due to the random shape parameter $a$ which creates round or ellipsoid shape. The ellipsoid/round shape does not evolve linearly with the parameter $a$. There are rounder shapes than ellipsoid ones among the 100 generated distributions. Sobel results are close to the full-vector gradient ones. It can be explained by color pairs oriented on the lightness axis. This theory is reinforced by the statement from Novak and Shafer [48] (cited by, for example [2], [6], [12]) stating that $90 \%$ of color edges can be processed in gray-level.

In order to better understand the results of table II, we present several cases of edges extraction in different cases of color distribution shapes and separability rates (fig. 10). Each column presents the segmentation results of the four presented gradients. The first row contains the images to segment.

The first column presents two green regions, the variations are mainly developed along one of the $R G B$ acquisition channels. Due to this particular distribution, the Di-Zenzo approach is well adapted. In addition, since color variations on the other axis are reduced, Sobel gradient also presents a good accuracy level.

In the second case, the color distribution is based on a pink color, so a not-saturated red, hence we are closer to the achromatic axis. Here, the channel combination is more crucial for the gradient extraction, even if we are theoretically in a easier case $(r=0.5)$. This necessity of an accurate color consideration explains the good accuracy of FVG versus the 
Table II

AVERAGE AND STANDARD DEVIATION OF ACCURACY FOR GIVEN SEPARABILITY RATE

\begin{tabular}{|r|c|c|c|c|c|c|c|c|}
\hline & \multicolumn{2}{|c|}{ Di Zenzo } & \multicolumn{2}{|c|}{ FVG } & \multicolumn{2}{c|}{ Sobel } & \multicolumn{2}{c|}{ Carron } \\
r & Mean & Std Dev & Mean & Std Dev & Mean & Std Dev & Mean & Std Dev \\
\hline-1 & 76.41 & 0.14 & $\mathbf{7 9 . 4 1}$ & 0.46 & 78.45 & 0.38 & 75.84 & 0.19 \\
\hline-0.5 & 78.57 & 0.30 & $\mathbf{8 3 . 1 7}$ & 0.62 & 81.36 & 0.61 & 76.97 & 0.25 \\
\hline-0.25 & 80.84 & 0.47 & $\mathbf{8 6 . 2 2}$ & 0.75 & 83.68 & 0.72 & 78.26 & 0.50 \\
\hline-0.15 & 82.17 & 0.66 & $\mathbf{8 7 . 7 0}$ & 0.77 & 84.75 & 0.84 & 78.96 & 0.56 \\
\hline 0 & 84.78 & 0.69 & $\mathbf{9 0 . 0 4}$ & 0.81 & 86.58 & 0.96 & 80.48 & 0.79 \\
\hline 0.15 & 88.02 & 0.74 & $\mathbf{9 2 . 4 6}$ & 0.84 & 88.62 & 1.07 & 82.66 & 0.85 \\
\hline 0.25 & 90.59 & 0.84 & $\mathbf{9 4 . 1 1}$ & 0.85 & 90.08 & 1.12 & 84.48 & 0.92 \\
\hline 0.5 & 96.58 & 0.66 & $\mathbf{9 7 . 5 5}$ & 0.75 & 93.31 & 1.09 & 90.94 & 0.66 \\
\hline
\end{tabular}

others

The results of the third column express the difficulty for the different approaches to work around the achromatic axis. In this case, the Sobel approach developed in gray-level color space is well adapted. We observe that the Carron approach, which includes an intensity axis, obtains the second score.

Finally, in the last case, the yellow distributions are far from the acquisition channels and from the achromatic axis. In this case, the necessity to correctly combine the color axis becomes important and explains the FVG score.

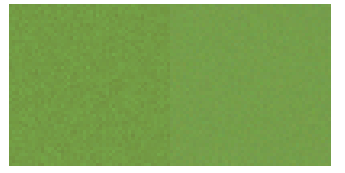

(a) $r=-0.5$

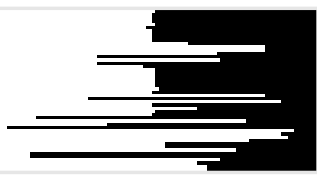

(e) Full Vector Gradient segmentation, $A c c=85 \%$

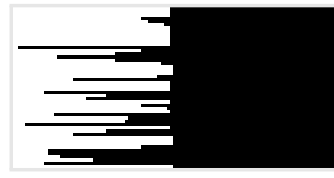

(i) $\mathrm{Di}$ Zenzo segmentation, $A c c=87 \%$

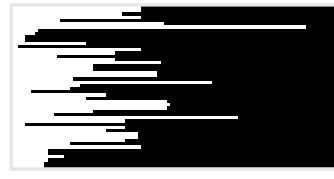

(m) Carron segmentation, $A c c=76 \%$

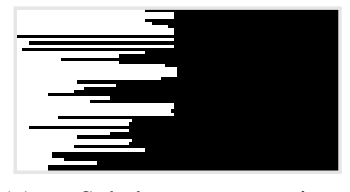

(q) Sobel segmentation, $A c c=81 \%$

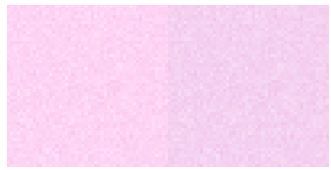

(b) $r=0.5$

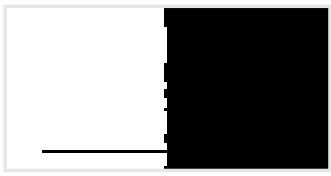

(f) Full Vector Gradient segmentation, $A c c=99 \%$

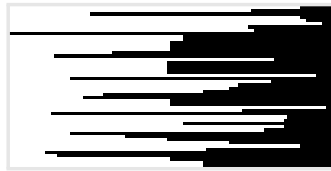

(j) Di Zenzo segmentation, $A c c=78 \%$

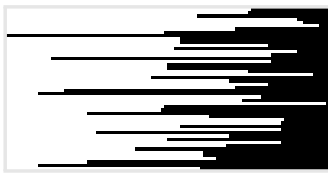

(n) Carron segmentation, Acc $=77 \%$

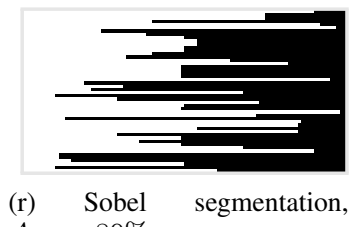
$A c c=80 \%$

\section{Subjective Comparison of Performance from Real Images}

To obtain an objective and valid comparison of performances requires image databases with known and accurate segmentation. We also need to know the color complexity of the problem to establish an associated separability rate. This is clearly not achievable with the existing multimedia databases. Thereafter, we will only subjectively compare the studied gradient approaches on some selected images from two databases. To reach color content expectations, we selected images from the natural world which present a sufficient color

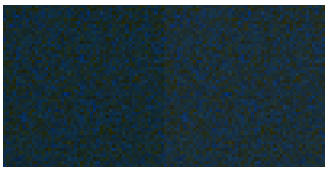

(c) $r=-1$

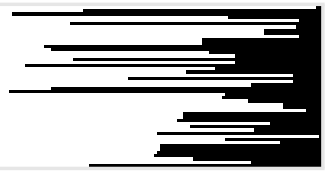

(g) Full Vector Gradient segmentation, $A c c=75 \%$

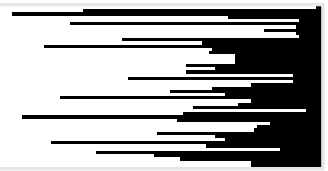

(k) Di Zenzo segmentation, $A c c=76 \%$

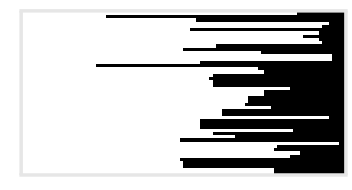

(o) Carron segmentation, Acc $=78 \%$

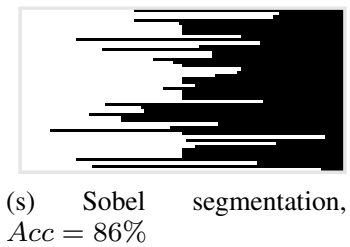

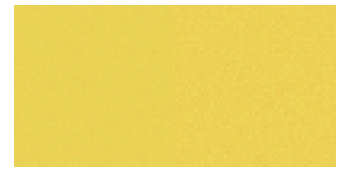

(d) $r=-0.5$

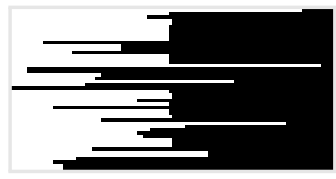

(h) Full Vector Gradient segmentation, $A c c=85 \%$

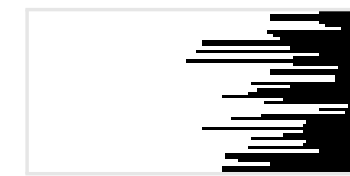

(1) Di Zenzo segmentation, Acc $=74 \%$

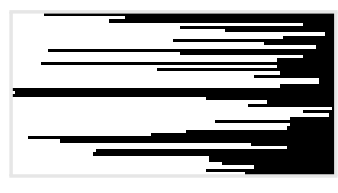

(p) Carron segmentation, Acc $=72 \%$

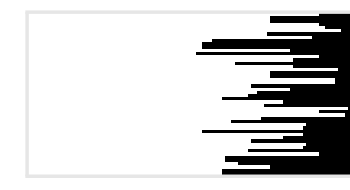

(t) Sobel Acc $=74 \%$

Figure 10. Examples of edge detection for images generated with $\Delta E=3$. Figures $10 \mathrm{a}, 10 \mathrm{~b}, 10 \mathrm{c}$ and $10 \mathrm{~d}$ are used to estimate gradients. Others present the segmentation results obtained, the accuracy results are given in the subtitle. 


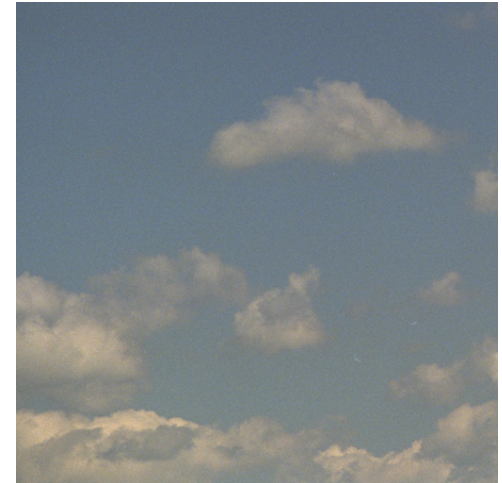

(a) Reference

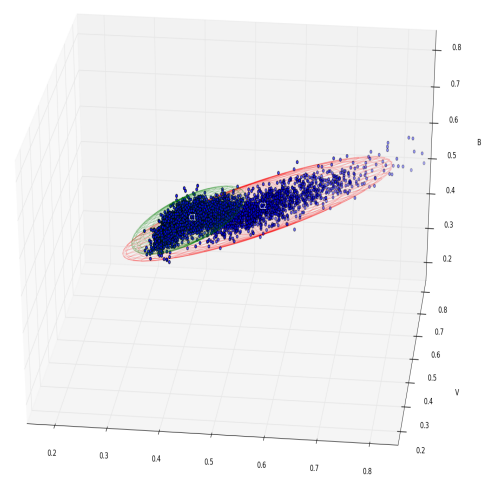

(d) Color histogram and a 2-class model

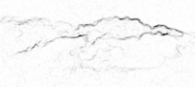

(b) Gray level Sobel gradient

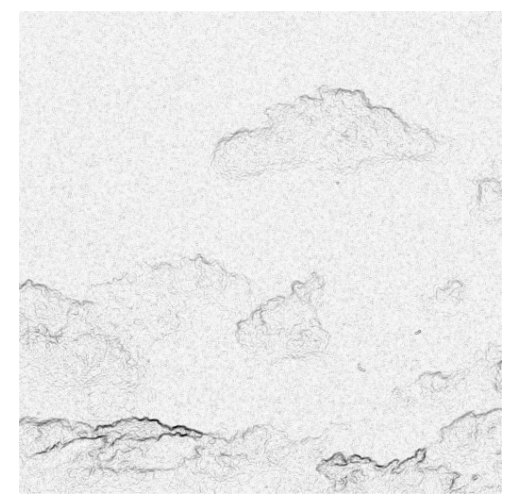

(e) Full vector gradient

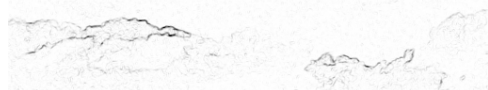

(c) Carron gradient

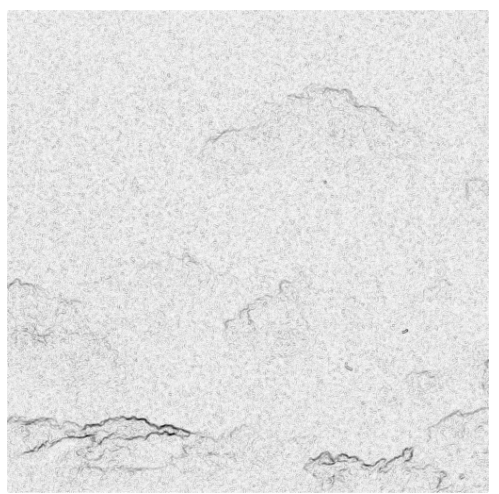

(f) Di Zenzo gradient

Figure 11. Gradient comparison of a cloud image (VisTex database). Figure 11d represents the color image histogram separated into two Gaussian distributions. These distributions give a separability rate of $r=-0.91$. Gradient images have been inverted.

complexity compared to man-made objects.

The first selected image (fig. 11a) is composed of clouds (gray to white) on a non-uniform blue sky. Sobel gradient computed from a gray level image or Carron's approach should give a good result. Unfortunately, they do not, even if color complexity seems weak, a more adapted color gradient is required. The image histogram (fig. 11d) explains these limits. Gaussian mixture model estimates two classes from the histogram. Both ellipsoids highly overlap. This model allows processing the separability rate: -0.91 . It explains why the Sobel, Carron and Di Zenzo approaches fail. Di Zenzo's image is noisier. Edges are missing on the cloud in the top and small clouds under it. The full-vector gradient extracts these edges correctly. Due to the high-level luminosity, channel values are important and nothing manages the interdependency between channels. Di Zenzo approach overestimates small differences in the blue sky and loses discrimination between diffuse clouds and sky. This result is fully consistent with synthetic data results.

The second image represents a flower from Stex database. This image is more chromatic, mainly in the orange, with a brown crown and some specular peaks due to water drops. Carron gradient produces better results than in the previous case, but its dynamic is weak (gradient histogram in fig. 12d). At first glance, Di Zenzo approach seems to propose more information. But the extracted dots correspond to the specular peaks observed in the flower center. These specular dots reduce the discrimination of pistils. Despite these differences, the two approaches produce similar results.

Figure 13 presents three results of edge extraction after segmenting the gradient image. The threshold applied is the third quartile of gradient histograms. Black pixels are edges detected by both gradients. Red pixels are edges detected by FVG but not by Di Zenzo. On the contrary, cyan pixels are edges detected by Di Zenzo and not by FVG. The three colors legend allows comparing both approaches.

On figure 13d (as fig. 12), edges from the full-vector gradient (black and red pixels) show the rounded part of the flower center and segment better the stamens on the left. On the contrary, Di Zenzo (black and cyan pixels) segments the shiny water.

On figure 13e, Di Zenzo approach overestimates the texture variation in the light wood stick. On the contrary, the fullvector gradient is more sensitive in dark regions.

Figure $13 \mathrm{f}$ is the most interesting. The flower center has a concentric shape construction, from brown to yellow, white and finally blue. On the bottom right flower (fig. 13f), Di Zenzo gradient highlights all the yellow pixels, while the fullvector gradient focuses on the white borders. The white center of the top right flower is better segmented by the full-vector gradient than Di Zenzo. We can say the full vector gradient gives better results than Di Zenzo. 


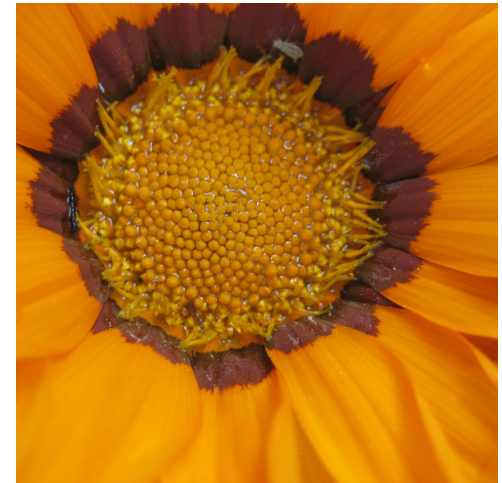

(a) Reference

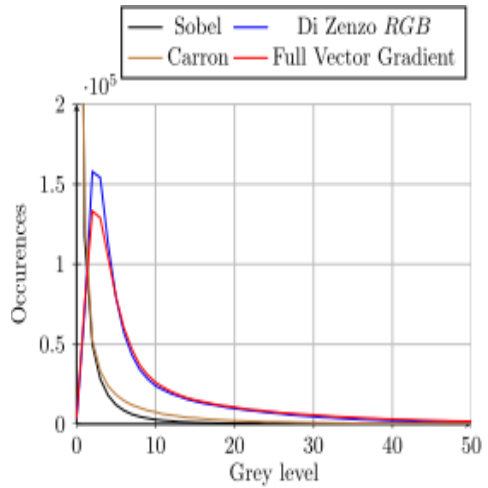

(d) Gradient's Histograms (b) Gray level Sobel gradient

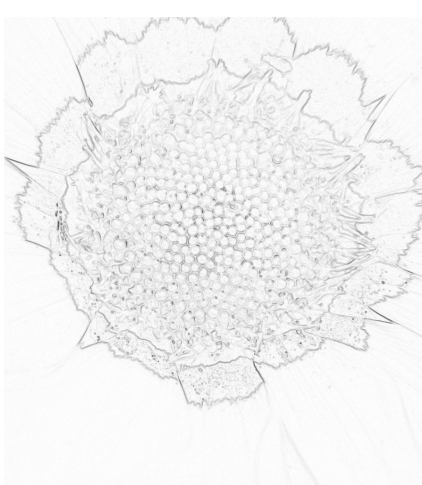

(e) Full vector gradient (c) Carron gradient

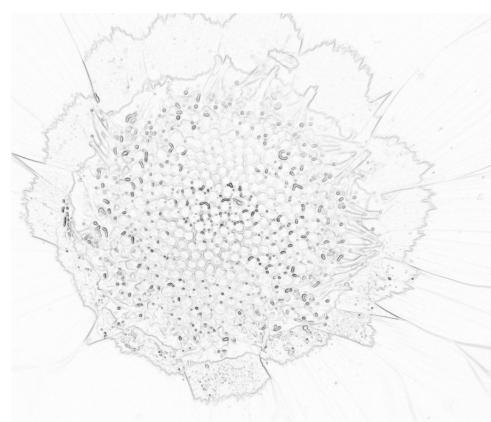

(f) Di Zenzo gradient

Figure 12. Gradient comparison of a flower image (Stex database). Figure 12d represents the gradient histograms. Gradient images have been inverted.

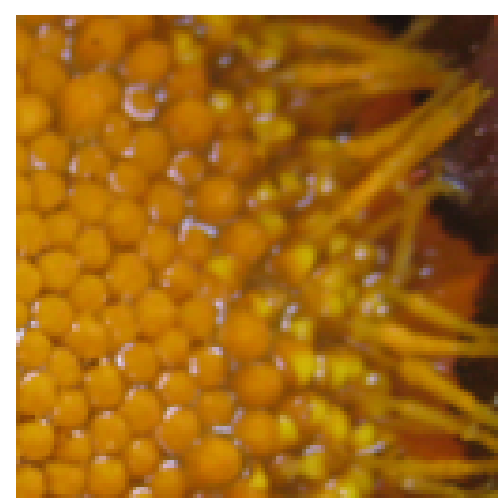

(a) Yellow flower

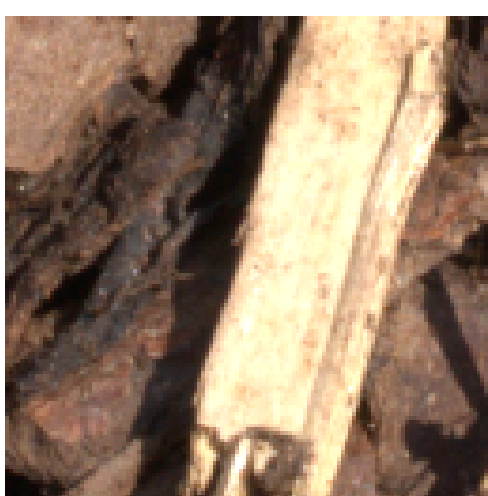

(b) Bark

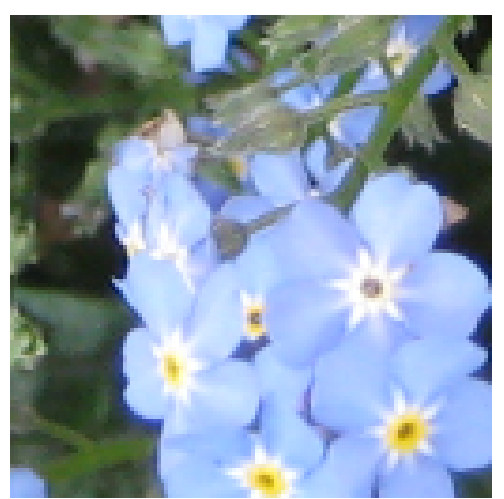

(c) Blue flower

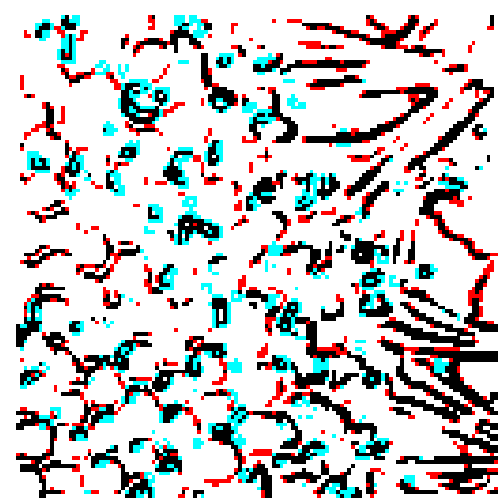

(d) Di Zenzo and full-vector gradient segmentation

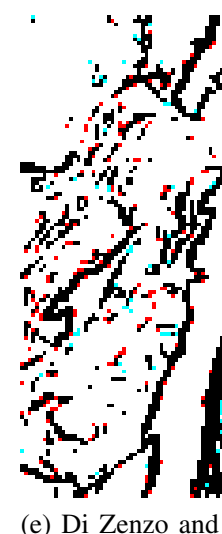

(e) Di Zenzo and full-vector gradient segmentation

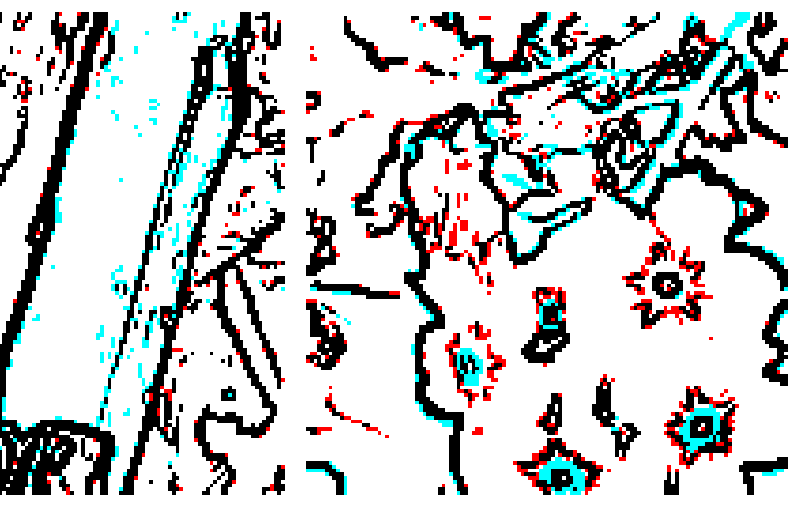

(f) Di Zenzo and full-vector gradient segmentation

Figure 13. Differences in edge extraction between FVG and Di-Zenzo approaches. The threshold is fixed to the third quartile of the image gradient histogram. Black pixels correspond to edges extracted by the two approaches, Cyan pixels are attributed when Di Zenzo approach extracts edges but not FVG and red pixels when FVG approach extracts edges but not Di Zenzo. 


\section{CONCLUSION}

In this article, we address the question of the gradient computation from multi-spectral images, where the acquisition space is not defined by independent axis. Such space configurations are due to the overlapping of sensors' spectral sensitivity curves. Under this definition, the trichromatic color sensor is considered as a multi-spectral sensor. Our work is dedicated to metrological purposes using color and spectral imaging, so the gradient is processed in the acquisition space.

Firstly, we have shown that the vectorial gradient proposed by Di Zenzo was implicitly defined for theoretical representation space based on orthogonal channels. Then, the fullvector gradient $(\mathrm{FVG})$ is defined embedding a complete scalar product between the spectral sensitivity curves of the sensor using the Gram matrix. This construction is fully generic, whatever the number or entanglement of the acquisition channels. Hence, Di Zenzo approach becomes a sub-case of the proposed construction for the trichromatic color acquisitions in the visible range. Results are provided for the same scene acquired in color (three channels) and using a multi-spectral snapshot sensor (nine channels).

Due to the lack of multi-spectral domain reference results of gradient extraction, the result performances are compared to the most-used approaches in the color domain: the classical Di Zenzo in the acquisition space $(R G B)$, the Carron gradient dividing the process into an $I H L S$ color space and the Sobel gradient in the equivalent gray-level image. Performances were assessed from generated images with controlled color distributions. When different color distributions are merged in the experimental test, the gain in performance reaches $6 \%$. Nevertheless, for specific color distributions, the gain is up to $15 \%$. This second case is closer to real applications in Control Quality by Vision. Gradient extraction comparison from real images reinforced the results obtained from the artificial dataset. The full-vector gradient is more sensitive to color variations than the Di Zenzo approach. In the presented cases, Sobel and Carron's approaches were unadapted to extract the expected edges.

In order to construct an objective test, we created controlled color distributions in the $C I E R G B$ color space. Nonetheless, for a complete validation under metrological constraints, a new dataset must be developed in order to produce physical samples for validation in and outside the visible range, but with fully controlled conditions of the pixel value distributions.

\section{ACKNOWLEDGEMENT}

This work has been supported by French national projects ANR DigiPi and CPER NUMERIC/e-Patrimoine supported by the New-Aquitaine region and Europe. We would also like to thank SILIOS Technologies for providing the data on the multispectral snapshot sensor SILIOS CMS-V.

\section{REFERENCES}

[1] S. Di Zenzo, "A note on the gradient of a multi-image," Computer vision, graphics, and image processing, vol. 33, no. 1, pp. 116-125, 1986.

[2] A. Koschan, "A comparative study on color edge detection," in Proceedings of the 2nd Asian Conference on Computer Vision, vol. 3, 1995, pp. 574-578.
[3] L. Luccheseyz and S. Mitray, "Color image segmentation: A state-ofthe-art survey," Proceedings of the Indian National Science Academy (INSA-A), vol. 67, no. 2, pp. 207-221, 2001.

[4] A. P. James, "Edge detection for pattern recognition: a survey," International Journal of Applied Pattern Recognition, vol. 3, no. 1, pp. 1-21, 2016.

[5] J. Canny, "A computational approach to edge detection," Pattern Analysis and Machine Intelligence, IEEE Transactions on, pp. 679-698, 1986.

[6] F.-P. Wang and P.-L. Shui, "Noise-robust color edge detector using gradient matrix and anisotropic gaussian directional derivative matrix," Pattern Recognition, vol. 52, pp. 346-357, 2016.

[7] C. I. Gonzalez, J. R. Castro, O. Mendoza, A. Rodríguez-Díaz, P. Melin, and O. Castillo, "Edge detection method based on interval type-2 fuzzy systems for color images," in Fuzzy Information Processing Society (NAFIPS) held jointly with 2015 5th World Conference on Soft Computing (WConSC), 2015 Annual Conference of the North American. IEEE, 2015, pp. 1-6.

[8] P. Shivakumara, D. Guru, and H. Basavaraju, "Color and gradient features for text segmentation from video frames," in Multimedia Processing, Communication and Computing Applications. Springer, 2013, pp. 267-278.

[9] M. J. Ehrhardt and S. R. Arridge, "Vector-valued image processing by parallel level sets," IEEE Transactions on Image Processing, vol. 23, no. 1, pp. 9-18, 2014.

[10] J. Astola, P. Haavisto, and Y. Neuvo, "Vector median filters," Proceedings of the IEEE, vol. 78, no. 4, pp. 678-689, 1990.

[11] P. E. Trahanias and A. N. Venetsanopoulos, "Color edge detection using vector order statistics," Image Processing, IEEE Transactions on, vol. 2, no. 2, pp. 259-264, 1993.

[12] A. N. Evans and X. U. Liu, "A morphological gradient approach to color edge detection," Image Processing, IEEE Transactions on, vol. 15, no. 6, pp. 1454-1463, 2006.

[13] E. Nezhadarya and R. K. Ward, "A new scheme for robust gradient vector estimation in color images," Image Processing, IEEE Transactions on, vol. 20, no. 8, pp. 2211-2220, 2011.

[14] P. Denis, P. Carre, and C. Fernandez-Maloigne, "Spatial and spectral quaternionic approaches for colour images," Computer Vision and Image Understanding, vol. 107, no. 1, pp. 74-87, 2007.

[15] J.-F. Rivest, P. Soille, and S. Beucher, "Morphological gradients," Journal of Electronic Imaging, vol. 2, no. 4, pp. 326-336, 1993.

[16] A. Ledoux and N. Richard, "Colour and multivariate texture features from vectorial mathematical morphology," Signal, Image and Video Processing (SIVP), Journal of, vol. 10, no. 3, pp. 431-438, 2016.

[17] A. Ledoux, N. Richard, and A.-S. Capelle-Laizé, "The fractal estimator: A validation criterion for the colour mathematical morphology," in Conference on Colour in Graphics, Imaging, and Vision. Society for Imaging Science and Technology, 2012, pp. 206-210.

[18] H.-C. Shih and E.-R. Liu, "Automatic reference color selection for adaptive mathematical morphology and application in image segmentation," IEEE Transactions on Image Processing, vol. 25, no. 10, pp. 4665-4676, 2016.

[19] H. Deborah, N. Richard, J. Y. Hardeberg, and C. Fernandez-Maloigne, "Assessment protocols and comparison of ordering relations for spectral image processing," IEEE Journal of Selected Topics in Applied Earth Observations and Remote Sensing, vol. 11, no. 4, pp. 1253-1265, 2018.

[20] M. Ruzon, C. Tomasi et al., "Edge, junction, and corner detection using color distributions," Pattern Analysis and Machine Intelligence, IEEE Transactions on, vol. 23, no. 11, pp. 1281-1295, 2001.

[21] Y. Deng, B. S. Manjunath, and H. Shin, "Color image segmentation," in Proc. IEEE Computer Society Conference on Computer Vision and Pattern Recognition CVPR'99, vol. 2, Fort Collins, CO, Jun. 1999, pp. 446-51.

[22] Z. Zareizadeh, R. P. Hasanzadeh, and G. Baghersalimi, "A recursive color image edge detection method using green's function approach," Optik-International Journal for Light and Electron Optics, vol. 124, no. 21, pp. 4847-4854, 2013.

[23] R. Bello-Cerezo, F. Bianconi, A. Fernández, E. González, and F. Di Maria, "Experimental comparison of color spaces for material classification," Journal of Electronic Imaging, vol. 25, no. 6, pp. $061406-$ $061406,2016$.

[24] X. Wang, R. Hänsch, L. Ma, and O. Hellwich, "Comparison of different color spaces for image segmentation using graph-cut," in Computer Vision Theory and Applications (VISAPP), 2014 International Conference on, vol. 1. IEEE, 2014, pp. 301-308.

[25] S. Wesolkowski, M. Jernigan, and R. D. Dony, "Comparison of color image edge detectors in multiple color spaces," in Image Processing, 
2000. Proceedings. 2000 International Conference on, vol. 2. IEEE, 2000, pp. 796-799.

[26] M. Dickinson, G. Bearman, S. Tille, R. Lansford, and S. Fraser, "Multispectral imaging and linear unmixing add a whole new dimension to laser scanning fluorescence microscopy," Biotechniques, vol. 31, no. 6, pp. 1272-1279, 2001.

[27] R. F. Lyon and P. M. Hubel, "Eyeing the camera: Into the next century," in Color and Imaging Conference, vol. 2002, no. 1. Society for Imaging Science and Technology, 2002, pp. 349-355.

[28] T. Carron and P. Lambert, "Color edge detector using jointly hue, saturation and intensity," in Image Processing, 1994. Proceedings. ICIP94., IEEE International Conference, 1994, pp. 977-981.

[29] T. Lei, Y. Fan, and Y. Wang, "Colour edge detection based on the fusion of hue component and principal component analysis," IET Image Processing, vol. 8, no. 1, pp. 44-55, 2014.

[30] A. Sáez, C. S. Mendoza, B. Acha, and C. Serrano, "Development and evaluation of perceptually adapted colour gradients," IET Image Processing, vol. 7, no. 4, pp. 355-363, 2013.

[31] J. Van de Weijer, T. Gevers, and J.-M. Geusebroek, "Edge and corner detection by photometric quasi-invariants," IEEE transactions on pattern analysis and machine intelligence, vol. 27, no. 4, pp. 625-630, 2005.

[32] R. Moreno, M. Grana, and A. d'Anjou, "An image color gradient preserving color constancy," in Fuzzy Systems (FUZZ), 2010 IEEE International Conference on. IEEE, 2010, pp. 1-5.

[33] J. Chu, J. Miao, G. Zhang, and L. Wang, "Edge and corner detection by color invariants," Optics \& Laser Technology, vol. 45, pp. 756-762, 2013.

[34] Z. Ying, G. Li, S. Wen, and G. Tan, "Orgb: Offset correction in rgb color space for illumination-robust image processing," in Acoustics, Speech and Signal Processing (ICASSP), 2017 IEEE International Conference on. IEEE, 2017, pp. 1557-1561.

[35] Y. Zhang, G. Wang, J. Xu, Z. Shi, and D. Dong, "The modified gradient edge detection method for the color filter array image of the cmos image sensor," Optics \& Laser Technology, vol. 62, pp. 73-81, 2014.

[36] A. Koschan and M. Abidi, "Detection and classification of edges in color images," IEEE Signal Processing Magazine, vol. 22, no. 1, pp. 64-73, 2005.

[37] L. G. Roberts, "Machine perception of three-dimensional solids," Ph.D dissertation, Massachusetts Institute of Technology, 1963.

[38] G. Sapiro, "Color snakes," Computer Vision and Image Understanding, vol. 68, no. 2, pp. 247-253, 1997.

[39] L. Jin, H. Liu, X. Xu, and E. Song, "Improved direction estimation for di zenzo's multichannel image gradient operator," Pattern Recognition, vol. 45, no. 12, pp. 4300-4311, 2012.

[40] Q. Wang, P. Li, W. Zuo, and L. Zhang, "Raid-g: Robust estimation of approximate infinite dimensional gaussian with application to material recognition," in 2016 IEEE Conference on Computer Vision and Pattern Recognition (CVPR), June 2016, pp. 4433-4441.

[41] S. K. Zhou and R. Chellappa, "From sample similarity to ensemble similarity: probabilistic distance measures in reproducing kernel hilbert space," IEEE Transactions on Pattern Analysis and Machine Intelligence, vol. 28, no. 6, pp. 917-929, June 2006.

[42] I. Sobel and G. Feldman, "A 3x3 isotropic gradient operator for image processing," the Stanford Artificial Intelligence Project, 1968.

[43] J. M. Prewitt, "Object enhancement and extraction," Picture processing and Psychopictorics, vol. 10, no. 1, pp. 15-19, 1970.

[44] A. Hanbury, "A 3d-polar coordinate colour representation well adapted to image analysis," in Scandinavian Conference on Image Analysis. Springer, 2003, pp. 804-811.

[45] B. Magnier, H. Abdulrahman, and P. Montesinos, "A review of supervised edge detection evaluation methods and an objective comparison of filtering gradient computations using hysteresis thresholds," Journal of Imaging, vol. 4, no. 6, p. 74, 2018.

[46] R. Kwitt and P. Meerwald, "Salzburg texture image database. available online: http://www.wavelab.at/sources/stex/."

[47] MIT, "Vision texture, vision and modeling group, cambridge. available online: http://vismod.media.mit.edu/pub/vistex/."

[48] C. Novak and S. Shafer, "Color edge detection," in Proc. DARPA Image Understanding Workshop, vol. 1, 1987, pp. 35-37.

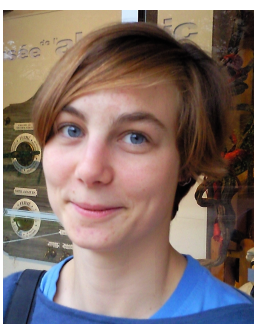

Hermine Chatoux is a Ph.D. student of the University of Poitiers. She is a member of the Icones Team in the XLIM institute (UMR CNRS 7252). She obtained an engineering degree in printing from INP Pagora school of Grenoble, France in 2007 and a master degree in applied mathematics in 2013 from the University Paul Sabatier in Toulouse, France. She is specialized in color, color perception and color management, her current research activities are merging mathematics and color sciences for color image similarity assessment in multimedia and cultural heritage domains.

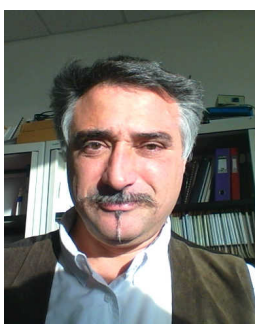

Noël Richard is an associate professor at the University of Poitiers as a researcher at XLIM laboratory, UMR CNRS 7552. He is the technical chair of the CIE Technical committee TC8.14 working on the definition and assessment of the spatio-chromatic complexity. Facing the lack of stability and accuracy of the existing approaches in color image processing, he developed a new image processing paradigm based on distance functions and non-linear expressions for metrological purposes. Since 2013, he is extending the paradigm to hyperspectral images, where a full-band vector processing is developing to respect the metrological constraints.

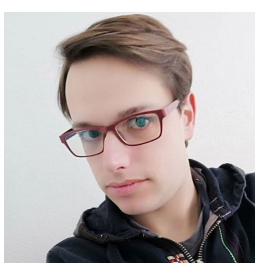

François Lecellier is an associate professor at the Institute of Technology of the University of Poitiers since 2010. He is a member of the Icones Team of the XLIM institute (UMR CNRS 7252). He obtained his Ph.D. in Computer Science in 2009 at the University of Caen (France). His research projects are in the domain of multimedia retrieval, multimedia databases, video copy detection and image processing. He also studies region-based active contours for image and video segmentation. Since 2017 he is the corresponding member of the working group Education Innovation of Coïmbra Group.

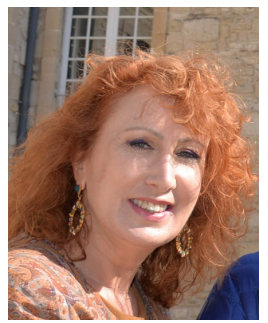

Christine Fernandez-Maloigne is currently ViceRector of Poitiers University, in charge of International Relations and director of a CNRS research federation (MIRES), which gathers 560 researchers in the south-west of France, in the area of mathematics, image processing, computer graphics, computer science and communication systems. Her research activities are focused on color imaging, including fundamental research about the introduction of human visual system models in multi-scale color image process as well as practical applications for biomedical, patrimonial and audiovisual digital contents. Christine FernandezMaloigne is now appointed member of the National Council of the French Universities (CNU), secretary of division 8, Image technologies, of the CIE (International Commission of Lighting) and deputy Editor-in-chief of JOSA A. 